1954

\title{
COUNCIL AND BOARD OF TRUSTEES
}

President...................................... T. WhyBURN

President Elect. . . . . . . . . . . . . . . . . . . . . . . . . . . R. L.

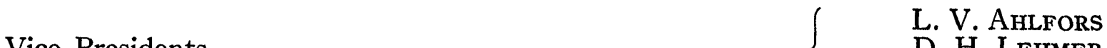

D. H. LEHMER

ANTONI ZYGMUND

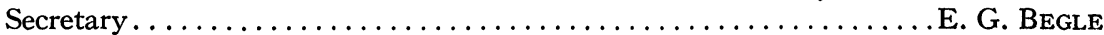

Associate Secretaries.......................... $\begin{array}{r}\text { L. W. CoHEN } \\ \text { J. W. GREEN }\end{array}$

J. H. ROBERTS

J. W. T. YOUNGS

Treasurer.................................. E. MEDER, JR.

Bulletin Editorial Committee................... $\{$ W. T. MARTiN

G. B. PRICE

RICHARD BRAUER

Proceedings Editorial Committee.................

A. C. SCHAEFFER

L. V. AhLFors

Transactions and Memoirs Editorial Committee.......... Herbert BUSEMANN ${ }^{1}$

J. L. Doob ${ }^{2}$

SAUNDERS MACLANE 3

Colloquium Editorial Committee.................. $\left\{\begin{array}{r}\text { A. A. ALBERT } \\ \text { EINAR HILLE }\end{array}\right.$

Deane Montgomery

Mathematical Reviews Editorial Committee........... $\left\{\begin{array}{r}\text { R. P. BoAS } \\ \text { EINAR HILLE }\end{array}\right.$

HASSLER WHITNEY

Mathematical Surveys Editorial Committee............ $\left\{\begin{array}{r}\text { MAx SHIFFMAN } \\ \text { R. J. WALKER }\end{array}\right.$ LEO ZIPPIN

Committee on Printing and Publishing

Representatives on Board of Editors of the American Journal of Mathematics.

M. R. HESTENES J. R. KLINE C. J. REES

REINHOLd BAER SAMUEL EILEN BERG ${ }^{4}$

To serve until

December, 1954

L. V. AhLFors

C. B. Allendoerfer

R. H. BING

E. R. LORCH

J. C. OXтову

H. F. Bohnenblust

B. P. GILL
Members at Large
To serve until
December, 1955
To serve until
December, 1956
F. B. JONES
E. E. Morse
B. J. Pettis
R. M. THRALL
G. W. WHITEHEAD

BOARD OF TRUSTEES
Einar Hille
J. R. KLINE
W. T. MARTIN

G. T. WHYBURN, ex officio

\footnotetext{
1 Replaced by Leo Z ippin for period September 1-December 31, 1954

2 Replaced by M. M. Day for period June 1 -November 10,1954 .

8 Replaced by O. F. G. Schilling for period June 1-November 10, 1954.

4 Replaced by E. R. Kolchin for period January-August, 1954.
} 